\title{
Effect of Microstructure on the Physical Properties of Cobalt-Base Alloys
}

\author{
KAMAL ASGAR and FLOYD A. PEYTON \\ University of Michigan, School of Dentistry, Ann Arbor, Michigan
}

Relatively little is known about the microstructure of cobalt-base alloys in dentistry. Manufacturers of dental cobalt-base alloys have published very little on this subject. No research has been conducted on the microstructure of the cobalt-base alloys used in dentistry. The need for research in this field is recognized, however, by both the manufacturers of the alloys and researchers in the dental field.

It appears that it would be profitable to dentistry if a study were made in which the effect of different casting conditions on the grain size and the microstructures of the cast product was established. It would also be useful to determine the physical properties of cast pieces having different microstructures. Today the mechanical properties listed for dental alloys vary a great deal from one type of alloy to another and also within the alloy. For example, elongation values from very small values to as high as 12 per cent are given for these alloys, and the modulus of elasticity determined for these alloys varies from approximately 20 to 40 million pounds per square inch. ${ }^{1,2}$ No satisfactory explanation is given for such a wide range of different physical properties. A study seems to be in order, therefore, to check these values and to determine whether such a variation is inherent in these alloys or is due to different testing conditions, such as sample size, casting condition, or equipment used. With these problems in mind, this study was undertaken in an effort to answer, with a certain amount of confidence, some of the above questions, the importance of which is becoming increasingly more obvious to the dental profession.

\section{MATERIALS AND METHODS}

In this study more than 120 specimens were cast under different conditions, and their physical properties as well as their microstructures were studied. All specimens were cast of a cobalt-base alloy* which is of a composition similar to a typical dental alloy. $\dagger$ The investment used was a phosphate-bonded type. $t$ Specimen size and manner of spruing were patterned after the recommendation of the A.D.A. specification for chromiumcobalt alloys. ${ }^{3}$ The conditions studied were related to changing the metal casting tem-

Presented before the Dental Materials Group of San Francisco, California, March, 1959.

This study was supported by a grant from the Office of Naval Research, Contract No. NR-180-360, to the University of Michigan, School of Dentistry.

Submitted by Kamal Asgar to the H. H. Rackham Graduate School of the University of Michigan as part of the thesis required for the Ph.D. degree.

Received for publication April 11, 1960; revised by authors August 18, 1960.

* Stellite 21.

† Vitallium.

$\$$ Ransom and Randolph No. 711 . 
perature, varying mold temperature, and heat-treating of the alloys. Melting and casting of the metal were carried out by using an induction casting machine.* The temperature of the molten metal in each melt was checked by means of an optical pyrometer. $\dagger$ The following four conditions were studied:

1. Molten metal was heated to $2,600^{\circ} \mathrm{F}$., then cast into a mold of $1,600^{\circ} \mathrm{F}$., and then allowed to cool on the bench to room temperature.

2. Molten metal was heated to $2,600^{\circ} \mathrm{F}$., cast into a mold of $1,300^{\circ} \mathrm{F}$., and benchcooled.

3. Molten metal was heated to $2,800^{\circ} \mathrm{F}$., cast into a mold of $1,600^{\circ} \mathrm{F}$., and benchcooled.

4. As in condition 1 , the metal was heated to $2,600^{\circ} \mathrm{F}$, cast into the mold of $1,600^{\circ} \mathrm{F}$, and bench-cooled. The castings then were heat-treated by placing them in an oven of $1,600^{\circ} \mathrm{F}$. for 1 hour and were then quenched in water.

After obtaining the physical properties of the cast specimens, one half of each specimen was imbedded in a plastic-type material for microstructure observations. In this way, the number of samples prepared for microstructure studies was equal to that for physical testing. Study of the microstructure at the fracture point rather than at any other point on the specimen was preferred. By knowing the microstructures at the fracture points, not only their general conditions could be studied, but also the cause of the fracture could be detected.

The results of the physical properties obtained in this study are reported separately. ${ }^{2}$

\section{RESULTS AND DISCUSSION}

The plastic material used to imbed the specimens formed a hard mass which did not smear during polishing. Although Stellite 21 is a hard alloy and does not smear during polishing and is not heat-treatable, it may be easily cold-worked. In polishing, special care should be exercised not to cold-work it excessively, and, by etching and polishing two or three times, any cold-worked surface may be removed. After being polished, the specimens were etched. According to Berglund, ${ }^{4}$ alloys which have special resistance to attack by chemical reagents, such as high stainless steels and nickel-chrome alloys, are best etched electrolytically. Since Stellite 21 falls in this category, the specimens were etched electrolytically. Different solutions, such as dilute phosphoric acid, 10 per cent solution of oxalic acid, 5 per cent solution of hydrochloric acid, and dilute solution of boric acid, were tried as electrolytes, with results varying from poor to fair. Following the recommendation of Faulkner ${ }^{5}$ at Haynes Stellite Company, a 2 per cent solution of chromic acid was used as an electrolyte, and the results were entirely satisfactory. Consequently, all the specimens were etched electrolytically, with low current produced by a 6-volt battery, using a 2 per cent solution of chromic acid as an electrolyte.

Stellite 21 does not have a simple microstructure, but instead it consists of an austenitic matrix, composed of the solid solution of cobalt and chromium as cast, and the matrix has a cored dendritic structure, as shown in Figure 1. According to Badger and Sweeney, ${ }^{6}$ the microstructure of Stellite 21 consists of three different types of carbide formations, as well as a eutectic and eutectoid structure similar to pearlite in formation. Three different types of carbide can be detected by using different types of etching solu-

* Williams Inductocast Unit.

† Leeds and Northrup. 
tions. If a very light electrolytic etch in 2 per cent chromic acid is used, followed by an alkaline permanganate etch of about 7 seconds, the different carbides will produce a selective staining action. One of the carbides, which contains both cobalt and molybdenum in solid solutions, had a brown stain, and the formula $\mathrm{Cr}_{4} \mathrm{C}$ was given to it. ${ }^{6} \mathrm{It}$ is believed that this carbide possesses a cubic structure. Another carbide with a chemical formula of $\mathrm{Cr}_{4} \mathrm{C}_{3}$ having a hexagonal structure also contains both cobalt and molybdenum in solid solution, and its color varies from very pale yellow to light tan. The third type has a general formula of $\mathrm{M}_{6} \mathrm{C}$, where $\mathrm{M}$ may be cobalt, chromium, or molybdenum, and this type of carbide reveals no definite structure. The color of this type of carbide

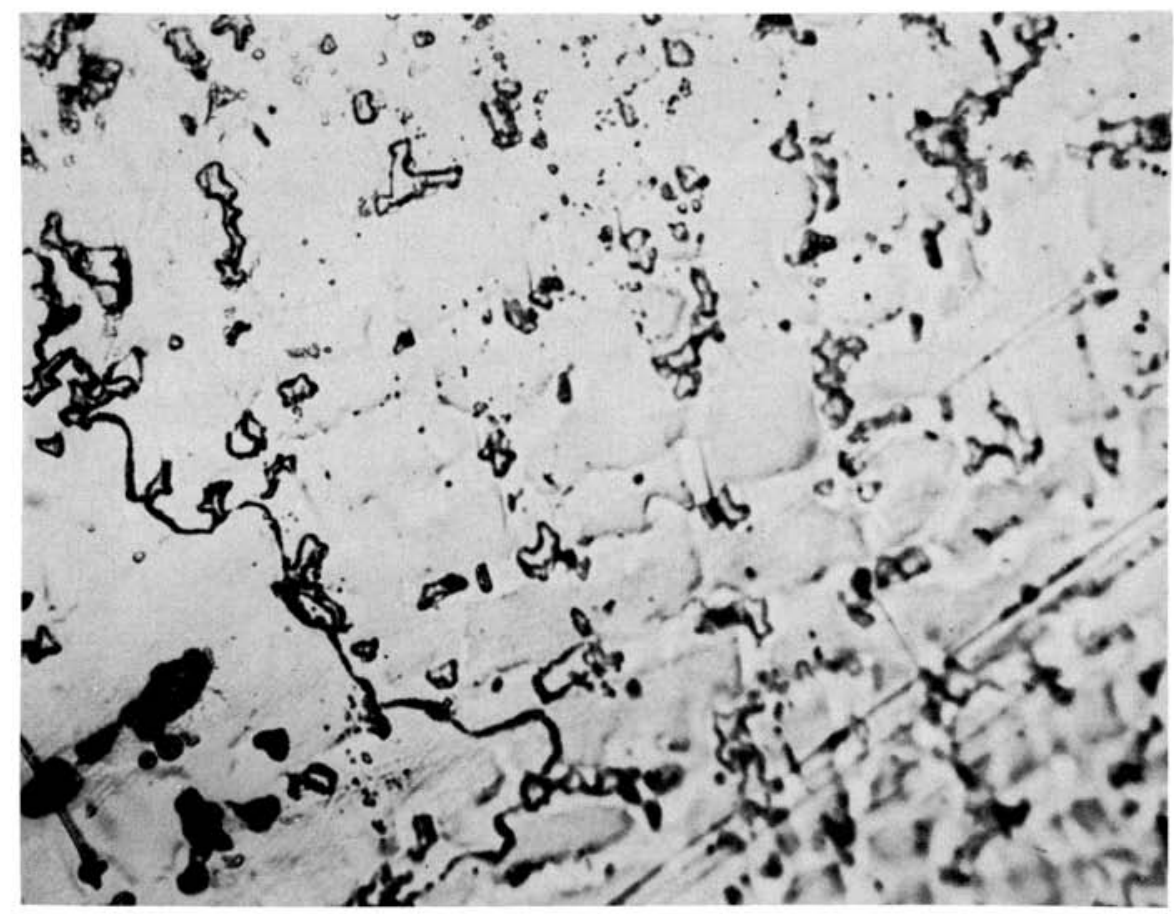

FIG. 1.-Microstructure of Stellite 21. (Mag. $\times 500$.)

may vary from red to green and occasionally from yellow to blue. In Figures 2 and 3 these three different types of carbides can be seen. The black-and-white photographs, however, obviously do not show the various colors obtained.

According to Grant, ${ }^{7}$ however, Badger and Sweeney's identification of the carbides is not complete, and the evidence is insufficient. Grant claims that the austenitic matrix of the alloy consists of perhaps two or three phases of carbides or a carbide of unknown composition.

It is beyond the scope of this study to check the composition of carbides and to accept either Grant's theory or the three different formulas for the carbides suggested by Badger and Sweeney. The purpose of this study, however, is to investigate the effect of the microstructure of a specimen on its physical properties. In general, regardless of the three types of carbides which may exist in any specimen, the microstructure of the alloy 


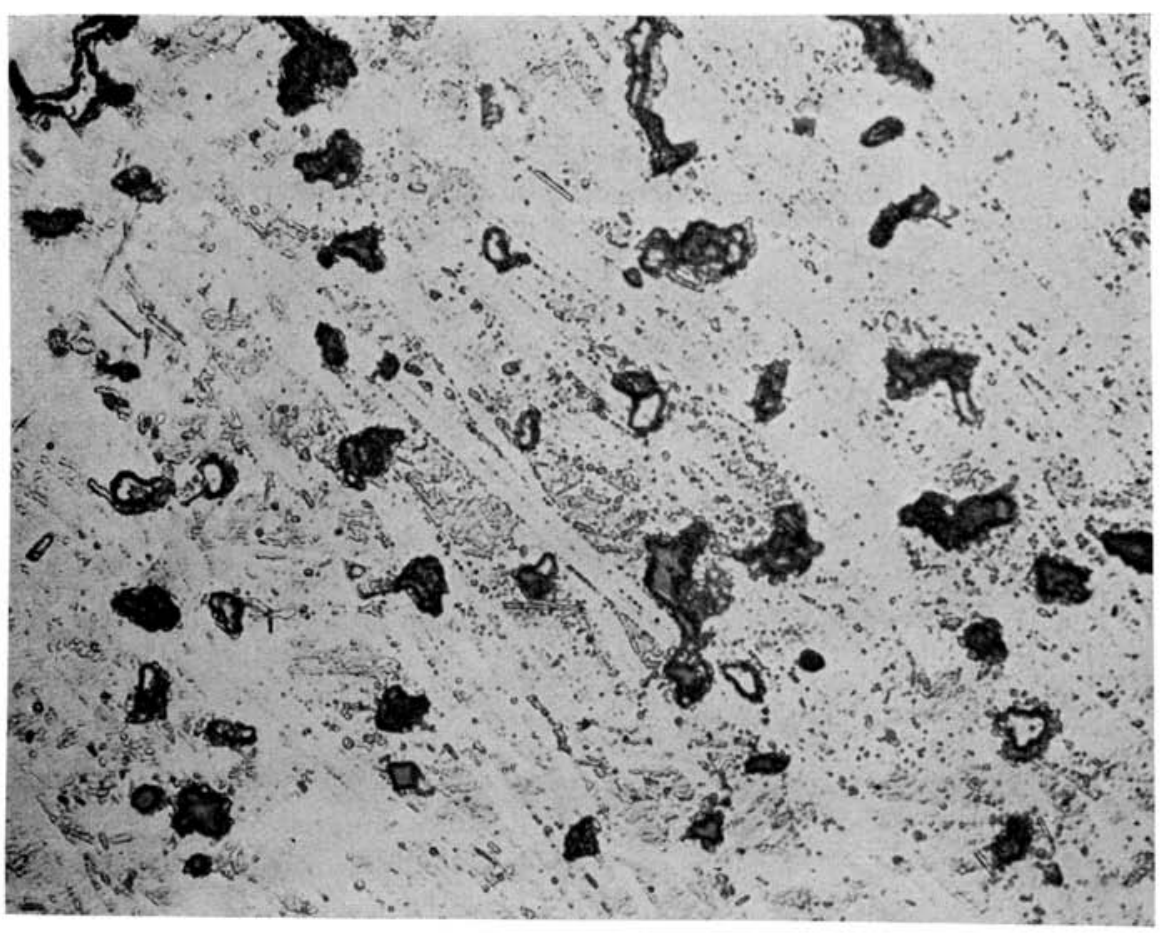

FIG. 2.-Microstructure of Stellite 21. (Mag. $\times 800$.) Different types of carbides

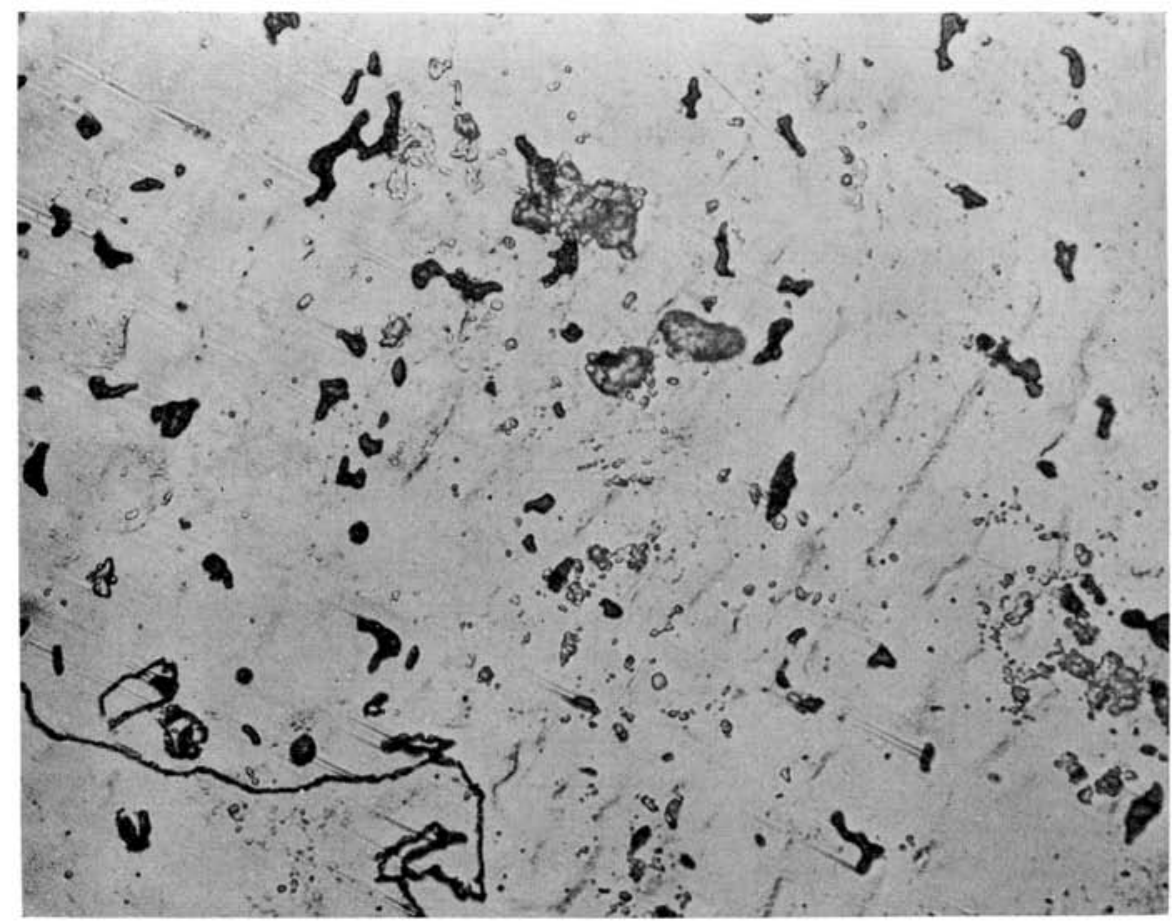

Fig. 3.-Microstructure of Stellite 21. (Mag. $\times 800$.) Different types of carbides 
under consideration may represent four distinct types: (1) carbides in the form of grain boundaries and continuous in nature, as shown in Figure 4; (2) carbides that are spherical and discontinuous like islands, as shown in Figure 5; (3) dark eutectoid areas which are present close to and along the grain boundaries, as shown in Figure 6; and (4) a large percentage of these eutectoid areas, which are lamellar in nature, as shown in Figure 7.

It was found that the presence or absence of any one of these four arrangements in the microstructure has a definite effect on the physical properties of the alloys. For example, Figure 8 is a photomicrograph of a representative structure in a series of twenty

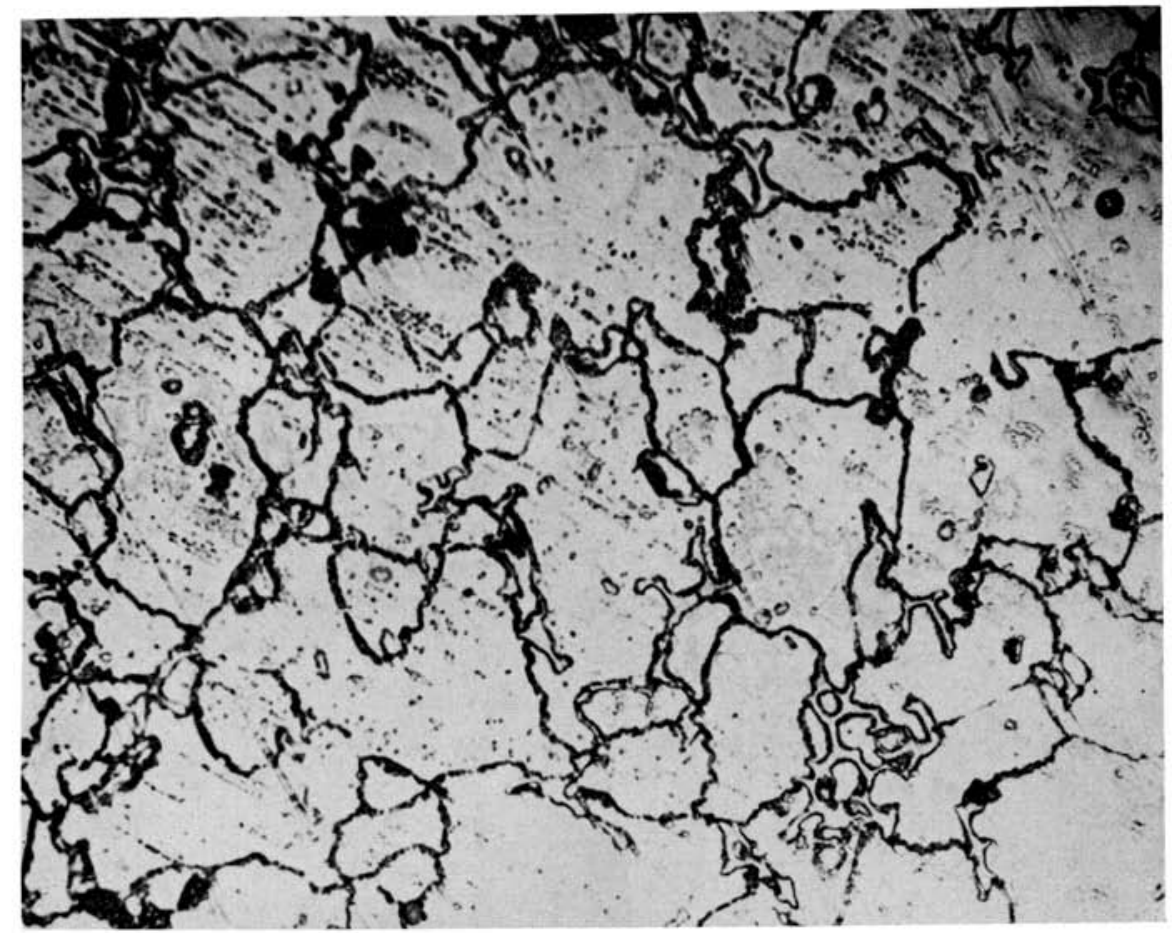

Fig. 4.-Microstructure of Stellite 21. (Mag. $\times 500$.) Continuous carbides

samples. In general, all the microstructures of this series showed many carbides forming within grain boundaries. This type of structure generally showed lower elongation values, and the average elongation of this group, which consisted of twenty cast specimens, was 3.4 per cent. Although all the specimens of this group were cast under similar conditions, for some unknown reason their microstructure was not alike in all specimens, nor did they have similar elongation values. It is interesting to note, however, that if, for any reason, they possessed a different type of microstructure from that in which the carbides formed within the grain boundaries, they also possessed a different value of elongation. For example, four of the twenty specimens of this group had elongations of $6.6,7.0,8.0$, and 7.2 per cent, which was much higher than the average elongation of only 3.4 per cent for the entire series of twenty samples. They also possessed a type of microstructure different from that representative of this group. In Figure 9 is shown 


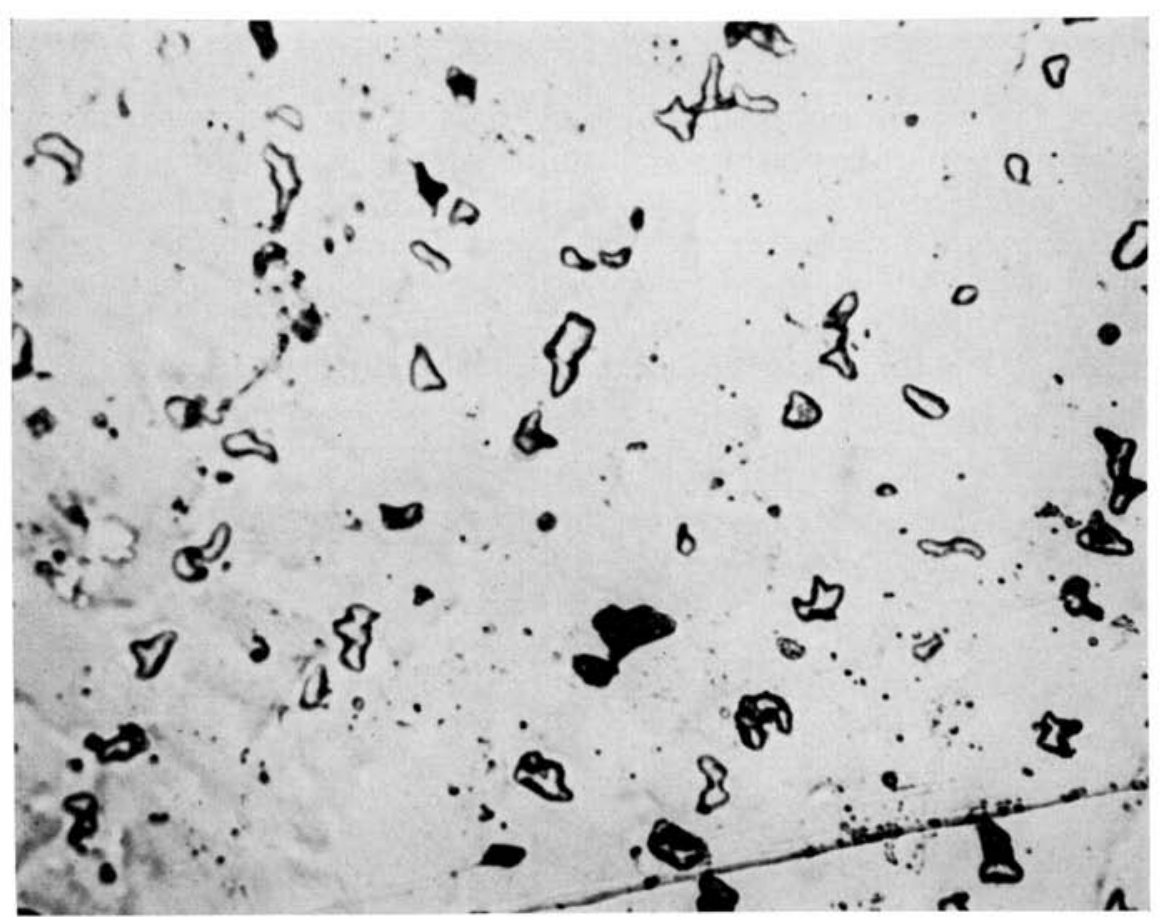

FIG. 5.-Microstructure of Stellite 21. (Mag. $\times 500$.) Spherical and discontinuous carbides

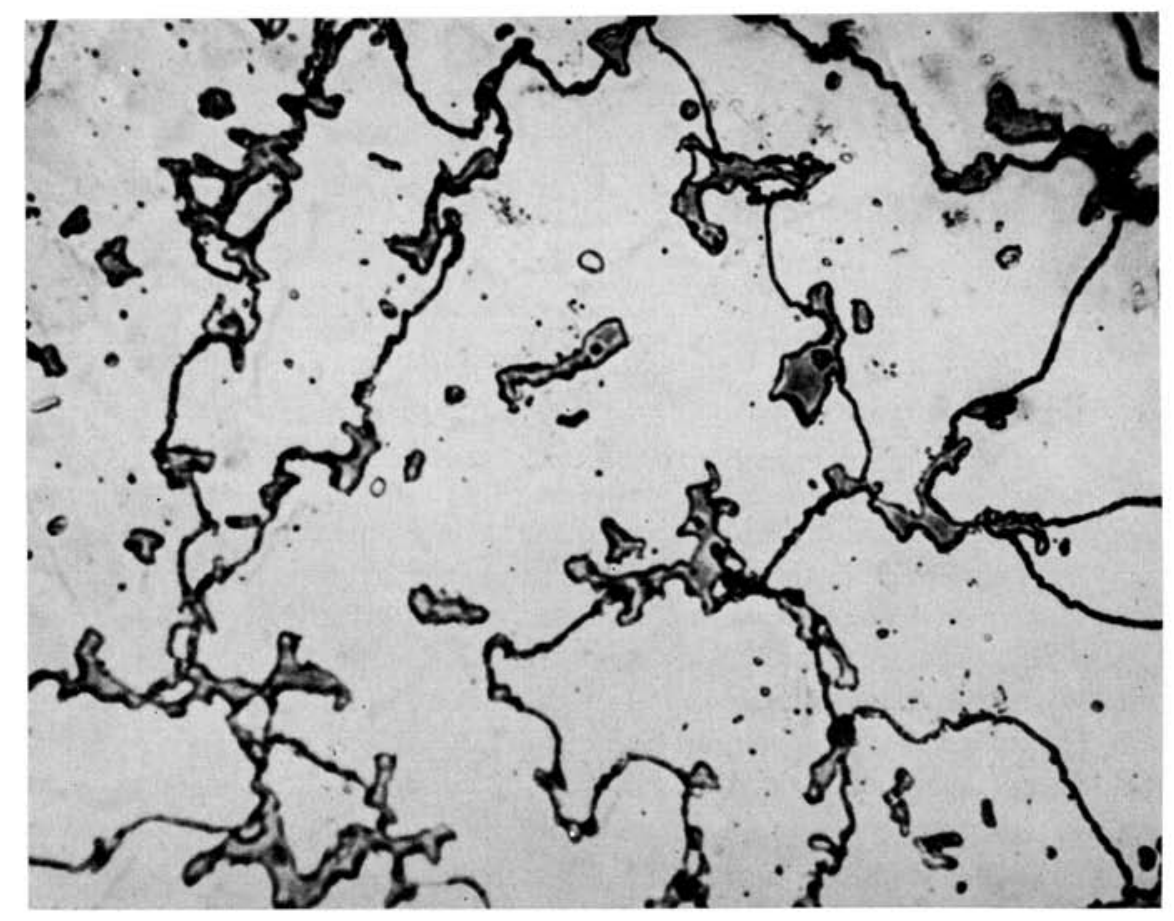

FIG. 6.-Microstructure of Stellite 21. (Mag. $\times 500$.) Dark eutectoid areas present close to grain boundaries. 


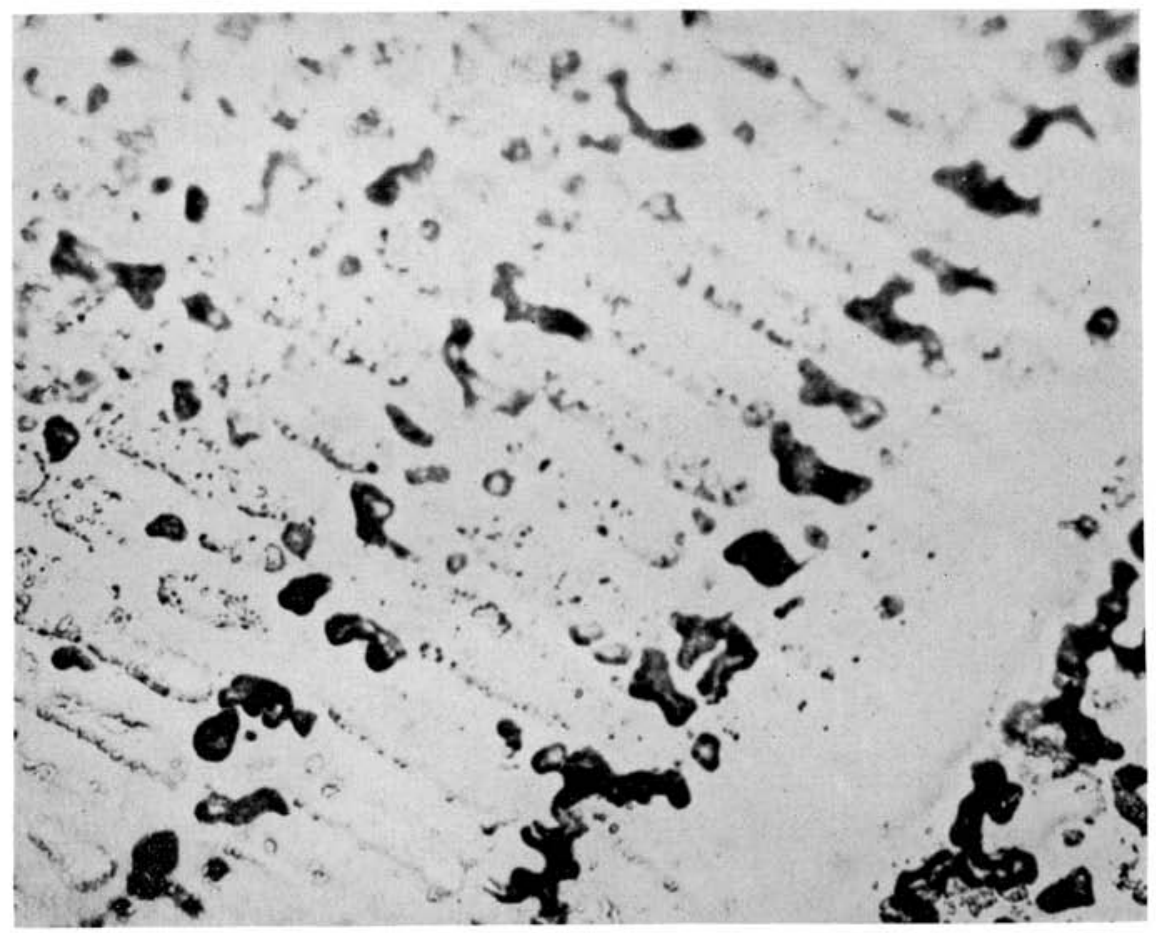

Fig. 7.-Microstructure of Stellite 21. (Mag. $\times 500$.) Larger percentage of entectoid areas lamellar in nature.

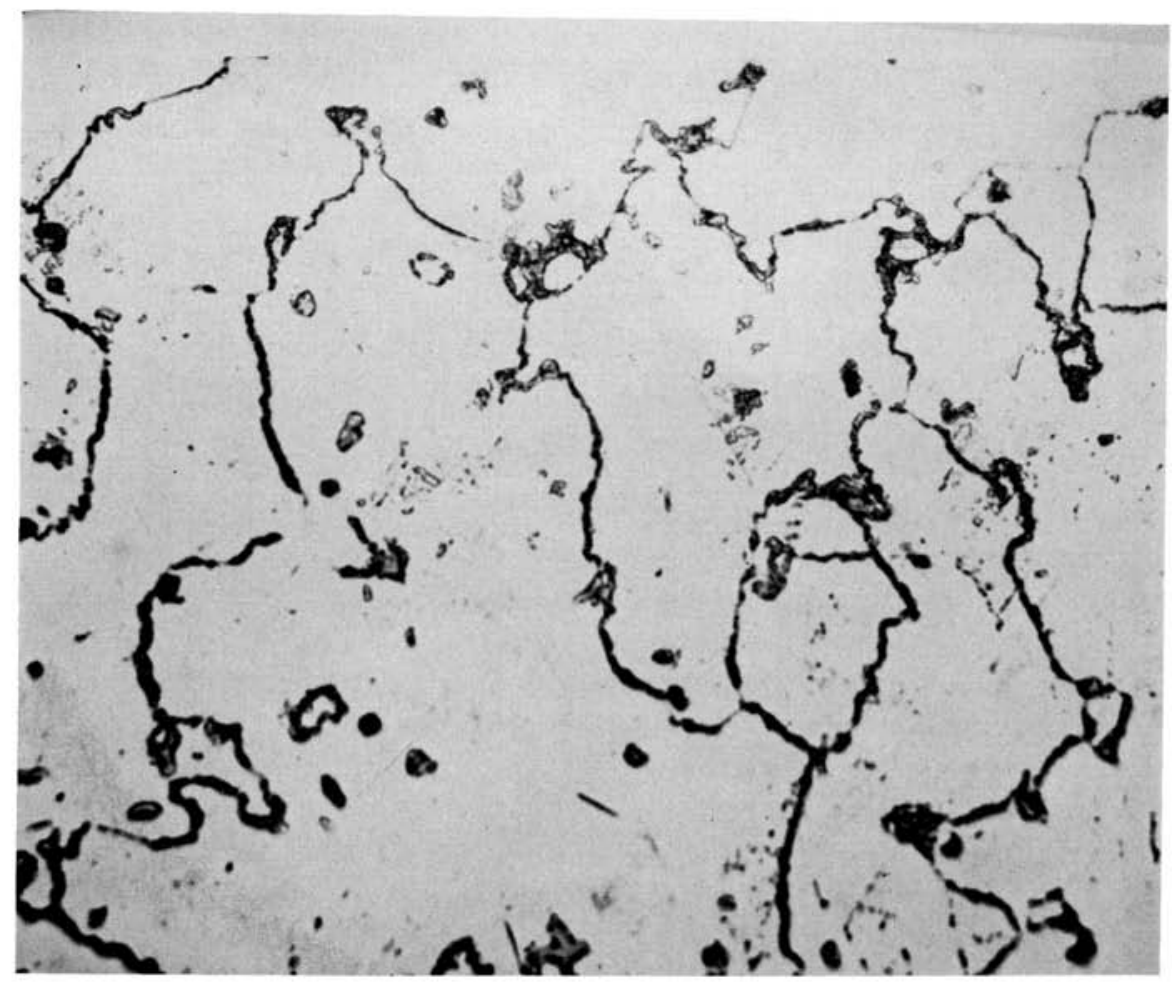

FIG. 8. - Microstructure of Stellite 21. (Mag. $\times 500$.) A typical structure of a series of twenty samples 
a typical photomicrograph of these four specimens, showing that these samples are different in microstructure as well as elongation from the other specimens in this series.

The spherical and discontinuous type of arrangement of carbides usually was obtained if the casting temperature of the molten metal was high. The elongation values of the specimens in this series were higher than for the specimens with continuous carbides, as discussed previously. Some of the specimens of this series with discontinuous carbides showed as much as 12 per cent elongation and a noticeable reduction in cross-sectional area of cast specimens. If any specimen with this type of microstructure showed lower elongation values, it was due to some other factors and not to its microstructure. One

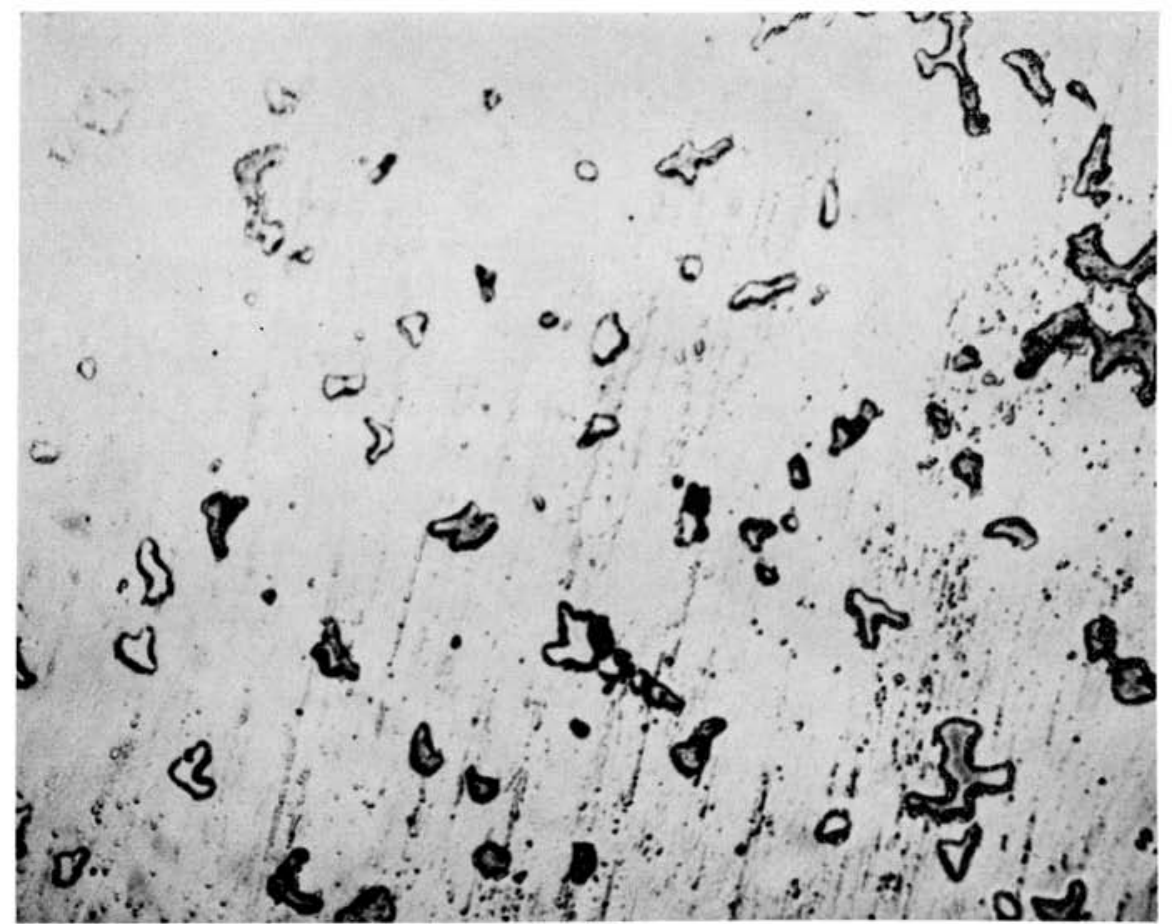

Fig. 9.-Microstructure of Stellite 21. (Mag. $\times 500$.) A representative structure of four specimens

reason for some of the lower elongation values of this series may have been the presence of microporosity. The brain boundary type of carbide occurred less frequently in this series of high casting temperatures, and the average number of grains was as low as two or three per cross-section.

Since the photomicrographs of the series with higher metal temperatures showed the spherical and discontinuous carbide arrangements, it was thought that some of the small amount of carbon present in the alloy might burn out during heating of the alloy to higher temperatures. In other words, a somewhat smaller amount of carbides seen in the micrographs of this series is thought to be due to the fact that the specimens cast under this condition may have ended by having less carbon and thus less carbides, which may have caused them to be discontinuous rather than continuous. To check this hypothesis, two specimens, one cast at $2,600^{\circ} \mathrm{F}$. and another cast at $2,800^{\circ} \mathrm{F}$., were pre- 
pared, and total carbon content was determined analytically. The results of this analysis showed that both specimens contained an equal amount, 0.23 per cent, carbon. These results agree with the results of a chemical analysis of the ingots made by the Haynes Stellite Company, in which the carbon content was reported to be 0.25 per cent. From these determinations, it can be observed that the total carbon content remains the same but that the carbide arrangement is different.

The presence of dark areas close to the grain boundaries, which Badger and Sweeney identified as eutectoid areas, may be detected in specimens cast under any one of the

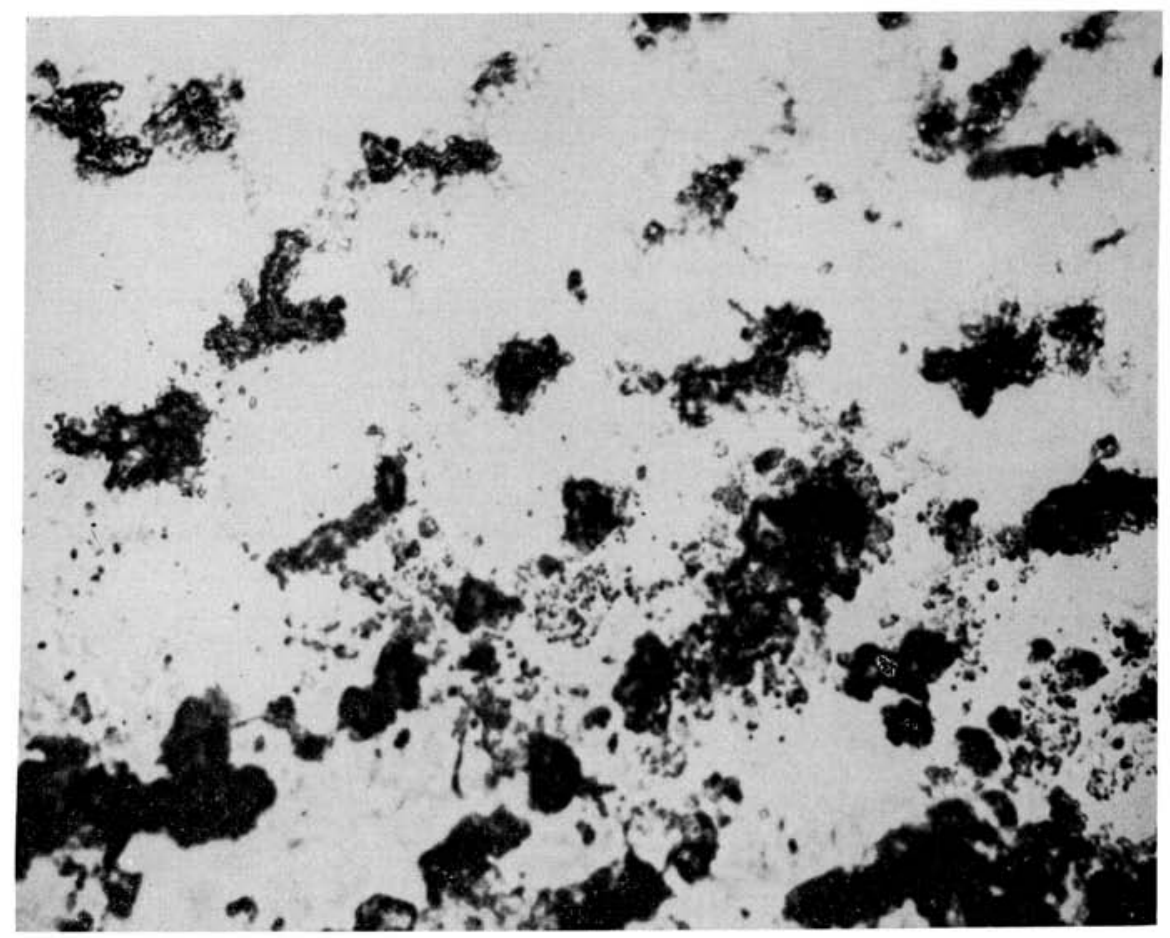

FIG. 10.-Microstructure of Stellite 21. (Mag. ×500.) Specimens were heat-treated; structure shows larger eutectoid areas.

conditions used in this study. This structure appears more frequently in the specimens cast at lower temperatures.

The micrographs of the specimens which were heat-treated showed larger eutectoid areas. These areas were lamellar in nature, as shown in Figure 10. The presence of this type of structure shows lower elongation and yield strength, as is shown in another report..$^{2}$

In this study it was found that, in order for the castings of this alloy to have higher elongation values, they not only should be sound and free from microporosity, but also their microstructure should be of a definite type. Spherical and discontinuous types of carbides which are free from eutectoid composition and microporosities possess greater ductility and elongation. Higher metal casting temperatures reult in such a microstructure. It is important, however, to note that as the metal casting temperature increases, 
the interphase reaction between the metal and the investment also increases. It should be remembered that the temperatures used in casting were $2,600^{\circ}, 2,800^{\circ}$, and $3,000^{\circ} \mathrm{F}$. Castings of $2,600^{\circ} \mathrm{F}$ had the best surface; with $2,800^{\circ} \mathrm{F}$, the metal was somewhat darker and less desirable. The surface of the castings of $3,000^{\circ} \mathrm{F}$. metal was very poor and practically unacceptable, but the specimens were more ductile, and the possibility of having microporosities was reduced considerably.

\section{SUMMARY}

1. Microstructure as well as microporosities have a pronounced effect on the physical properties of Stellite 21.

2. Stellite 21 can be etched satisfactorily with a 2 per cent solution of chromic acid as an electrolyte and a low current produced by a 6-volt battery.

3. According to Badger and Sweeney's identification, three different types of carbide can be detected in this alloy by using a very light electrolytic etch in 2 per cent chromic acid followed by an alkaline permanganate etch of about 7 seconds.

4. Carbides that are spherical and discontinuous, like islands, demonstrate the highest elongation. The carbides in the form of grain boundaries which are continuous or dark eutectoid areas reduce the elongation of this alloy.

5. The temperature of the mold has little effect on the microstructure. High metal casting temperatures, however, results in spherical and discontinuous carbides, which, in turn, yield higher elongation values.

\section{REFERENCES}

1. Taylor, D. F. Leibrfita, W. A., and Alder, A. G. Physical Properties of Chromium-Cobalt Alloys, J.A.D.A., 56:343, 1958.

2. Asgar, K., and Peyton, F. A. Effect of Casting Conditions on Some Mechanical Properties of Cobalt-Base Alloys, submitted to J. D. Res., March, 1960.

3. Taylor, D. F., and Sweeney, W. T. A Proposed Specification for Dental Chromium-Cobalt Casting Alloys, J.A.D.A., 54:44, 1957 .

4. Berglund, T. Metallographer's Handbook of Etching, p. 89. London and New York: Sir I. Pitman \& Sons, Ltd., 1931.

5. Faulkner, W. H., Haynes Stellite Company, Kokomo, Indtana. Personal communications, November, 1958.

6. BADGER, F. S., and SweEney, W. O. Metallurgy of High Temperature Alloys Used on Current Gas Turbine Designs: Symposium on Material for Gas Turbine, p. 99. Philadelphia: American Society for Testing Materials, 1946.

7. Grant, N. J. The Effect of Composition and Structural Changes on the Rupture Properties of Certain Heat Resistant Alloys at $1500^{\circ}$ F., Trans. Am. Soc. Metals, 39:368, 1747. 